\title{
BMJ Open Prescribing and deprescribing antihypertensive medication in older people by Dutch general practitioners: a qualitative study
}

\author{
Tessa van Middelaar, ${ }^{1,2}$ Sophie D Ivens, ${ }^{3}$ Petra G van Peet, ${ }^{4}$ \\ Rosalinde K E Poortvliet, ${ }^{4}$ Edo Richard, ${ }^{1,2}$ A Jeannette Pols, ${ }^{3}$ \\ Eric P. Moll van Charante ${ }^{3}$
}

To cite: van Middelaar T, Ivens SD, van Peet PG, et al. Prescribing and deprescribing antihypertensive medication in older people by Dutch general practitioners: a qualitative study. BMJ Open 2018;8:e020871. doi:10.1136/ bmjopen-2017-020871

- Prepublication history for this paper is available online. To view these files, please visit the journal online (http://dx.doi. org/10.1136/bmjopen-2017020871).

Received 28 November 2017 Revised 8 March 2018 Accepted 22 March 2018
Check for updates

${ }^{1}$ Department of Neurology, Institute for Brain, Cognition and Behaviour, Radboud University Medical Center, Nijmegen, The Netherlands

${ }^{2}$ Department of Neurology, Academic Medical Center (AMC), Amsterdam, The Netherlands ${ }^{3}$ Department of General Practice, Amsterdam Public Health research institute, Academic Medical Center (AMC), Amsterdam, The Netherlands

${ }^{4}$ Department of General Practice, Leiden University Medical Center (LUMC), Leiden, The Netherlands

Correspondence to Dr Tessa van Middelaar; t.vanmiddelaar@amc.uva.nl

\section{ABSTRACT}

Objectives To explore general practitioners' (GPs) routines and considerations on (de)prescribing antihypertensive medication (AHM) in older patients, their judgement on usability of the current guideline and needs for future support.

Design Semistructured interviews.

Setting Dutch general practice.

Participants Fifteen GPs were purposively sampled based on level of experience and practice characteristics until saturation was reached.

Results GPs appeared reluctant to start AHM, especially in patient $>80$ years. High systolic blood pressure and history of cardiovascular disease or diabetes were enablers to start or intensify treatment. Reasons to refrain from this were frailty and patient preference. GPs described a tendency to continue AHM regimens unchanged, influenced by daily time constraints, automated prescription routines and anticipating discomfort when disturbing patients' delicate balance. GPs were only inclined to deprescribe AHM in terminally ill patients or after prolonged achievement of target levels in combination with side effects or patient preference. Deprescription was facilitated when GPs had experience with patients showing increased quality of life after deprescription and was withheld by anticipated regret (ie, GPs' fear of a stroke after deprescribing). GPs felt insufficient guidance from current guidelines, especially on deprescription.

Conclusions GPs are reluctant to start or deprescribe AHM in older people and have a propensity to continue AHM within a daily routine that insufficiently supports critical medication review. (De)prescription is influenced by patient preferences and anticipated regret and current guidelines provide insufficient guidance.

\section{INTRODUCTION}

Eighty per cent of older people with hypertension use antihypertensive medication (AHM). ${ }^{1}$ However, treatment recommendations for people aged 65 and over are limited and there is considerable variation in treatment policy between general practitioners
Strengths and limitations of this study

- This is the first qualitative study on general practitioners' (GPs) reasons for prescription and deprescription of antihypertensive medication in older patients.

- A broad diversity in GPs, varying in experience and setting, were interviewed because of our purposive sampling method.

- The interviewed GPs often came to new insights and ideas during the interview, when provided with ample time to reflect on decisions that they had made.

- Only Dutch GPs were included, potentially limiting the generalisability of our results to other general practice settings.

- Relative few GPs gave examples were antihypertensive medication was started, potentially limiting the scope of reasons to start.

(GPs). ${ }^{23}$ The European guideline on cardiovascular disease (CVD) prevention states that in this older population AHM is still effective, but that for people over 80 years old target systolic blood pressure (SBP) levels may be less strict (eg, 140-150 mm $\mathrm{Hg}$ instead of $<140 \mathrm{~mm} \mathrm{Hg}){ }^{2}$ No recommendations are provided on deprescription (ie, decreasing dosage or discontinuing).

In the past, older people were mostly excluded from clinical trials on AHM and cardiovascularprevention. More recentstudies that have included this group have presented conflicting results. Overall, antihypertensive treatment in the oldest old ( $>80$ years) seems to reduce cardiovascular morbidity but has no effect on overall mortality. ${ }^{4}$ The Systolic Blood Pressure Intervention Trial (SPRINT) provided evidence that intensive BP control to a target SBP of below $120 \mathrm{~mm} \mathrm{Hg}$ could prevent CVD and mortality, also in people aged $\geq 75$ years. ${ }^{56}$ This trial also showed that 
a low target SBP did not result in more orthostatic hypotension, falls or acute kidney injury, which are concerns regarding intensive BP treatment. ${ }^{7}$ However, it remains unclear to what extent these results are generalisable. ${ }^{89}$ On the other end of the spectrum, observational studies have shown that in frail people $>80$ years old a BP below $140 / 90 \mathrm{~mm} \mathrm{Hg}$ might even be harmful as it is associated with an increased mortality risk. ${ }^{10}$ In these oldest old people, it may therefore be recommended to restrict to two antihypertensive drugs and aim for a maximum reduction in SBP of $15 \mathrm{~mm} \mathrm{Hg} .{ }^{40}$ This, however, is not included in the current guidelines.

Our primary aim was to explore GPs' routines and considerations on prescribing and deprescribing AHM in older people to clarify the processes underlying current (de) prescribing practices. Our secondary aim was to assess GPs' judgement on usability of current guideline and their needs for future support in this decision-making process to help improve future guidelines on antihypertensive treatment in older people.

\section{METHODS}

\section{Participants}

Between October 2015 and December 2016, 15 semistructured interviews were performed. The study was granted a waiver from ethical approval by the Medical Ethics Committee of the Academic Medical Center (AMC) in Amsterdam. All participants gave written informed consent. GPs from different geographical areas in the Netherlands were invited by telephone or email to participate in the interviews. Participants were purposively sampled based on years of experience, setting (rural or urban) and type of practice (small-scale vs healthcare centre), presence of a practice nurse and expertise with regard to cardiovascular risk management. Of the 44 GPs approached, 15 agreed to participate, 13 could not be reached and 16 refused participation. Main reason GPs declined to participate was time constraint. After 15 interviews, saturation was reached as no important new (sub) themes emerged over the last interviews.

\section{Data collection}

Each interview was done by one of two researchers (TvM and SI), both young female physicians trained in qualitative interviewing. The interviewers and interviewees had no prior relationship and they were aware of each other's profession and level of experience. A prepiloted

\section{Box 1 Main topics in the interview guide}

- Start or increase antihypertensive medication (AHM): patient from daily practice.

- Continue AHM: patient from daily practice.

- Deprescribe AHM: patient from daily practice.

- Current guideline.

- Needs and wishes for future guideline support. semistructured interview guide (box 1) was developed focused on behaviour and attitude in daily clinical practice to make the interview as concrete as possible and limit the influence of socially desirable answers. ${ }^{11}$ First, participating GPs were asked to retrieve recent case histories on antihypertensive treatment in older patients. They were primed for cases in which they recently started, increased, continued (without changes), decreased or discontinued AHM and were asked to elaborate on their routines and considerations in the treatment decision. It was left to the discretion of the GPs to decide who they considered an older patient. They were allowed to consult their electronic health record for further details. Second, their views on the value of the current guideline and needs for future support were discussed. The interviews were held in person at a place that was convenient for the interviewed GP (at their practice or home or at the AMC or Radboud University Medical Center). The interviews took approximately $45 \mathrm{~min}$, were audio-recorded and transcribed by the interviewer verbatim.

\section{Data analysis}

Transcripts were thematically analysed following an inductive and iterative approach. ${ }^{12}$ Two researchers (TvM and SI) independently coded all transcripts. After every two to four interviews, the researchers discussed the codes and through comparison and discussion a common coding system was developed. The (sub) themes derived from the codes were subsequently organised within the prespecified structure on AHM (de)prescription: starting, intensifying, continuing and deprescribing. At multiple times during the data collection and analysis phase, results were discussed among a team including a practising GP and an expert in qualitative research. Based on these discussions, the interview guide was adapted by adding reflective questions to study the contrast between routine behaviour and conscious considerations. Also, the tilting point for (de) prescription was further specified and the reasons for starting AHM were further explored as this is under-represented at the start.

\section{Patient and public involvement}

By exploring GPs' routines and views, we aim to describe daily clinical practice and the support necessary to improve clinical practice from a GP's perspective.

\section{RESULTS}

Fifteen GPs were interviewed varying in years of experience from 1 to 30 years (table 1 ). In daily practice, (de)prescription of AHM was consciously evaluated when practice nurses noticed a high BP in patients with a history of CVD or when patients actively approached their GP for their BP, had their BP measured for other reasons, for example when moving to an elderly home or underwent a critical review on polypharmacy in collaboration with the pharmacist. In table 2, the main barriers and enablers that GPs mentioned when starting, 


\begin{tabular}{|c|c|}
\hline Characteristic & Population $(n=15)$ \\
\hline \multicolumn{2}{|l|}{ Sex } \\
\hline Male & $8(53 \%)$ \\
\hline \multicolumn{2}{|l|}{ Age } \\
\hline$<40$ years & $7(47 \%)$ \\
\hline 40-50years & $3(20 \%)$ \\
\hline$>50$ years & $5(33 \%)$ \\
\hline \multicolumn{2}{|l|}{ Years as a GP } \\
\hline $0-5$ years & $4(27 \%)$ \\
\hline $5-10$ years & $3(20 \%)$ \\
\hline 10-15years & $3(20 \%)$ \\
\hline$>15$ years & $5(33 \%)$ \\
\hline \multicolumn{2}{|c|}{ Academically affiliated7 (47\%) } \\
\hline \multicolumn{2}{|l|}{ Location } \\
\hline Urban & $8(53 \%)$ \\
\hline Rural & $7(47 \%)$ \\
\hline \multicolumn{2}{|l|}{ Practice type } \\
\hline Solo & $2(13 \%)$ \\
\hline Duo & $4(27 \%)$ \\
\hline Group & $8(53 \%)$ \\
\hline Other† & $1(7 \%)$ \\
\hline Practice nurse available & $10(67 \%)$ \\
\hline
\end{tabular}

Characteristics of the participating general practitioners.

*Academically affiliated indicates either working at an academic centre for educational or research purpose or working as GP trainer.

†One GP worked as locum GP in different practices.

GP, general practitioner.

intensifying, continuing and deprescribing AHM are presented, illustrated by four cases (table 3). These barriers and enablers are further explained in the text below.

\section{Starting treatment}

In general, GPs were somewhat reluctant to start AHM in older patients. This was especially true for the oldest old patients ( $>80$ years), for patients with a limited life expectancy ( $<1-2$ year) and for frail patients. In these cases, GPs only started AHM when SBP was higher than $180 \mathrm{~mm} \mathrm{Hg}$. GPs evaluated frailty based on their clinical impression and physical, cognitive and overall functioning. One GP was cautious because of the psychological impact starting medication could have on patients:

Sometimes I think that the added value (of starting AHM) in patients at older ages is minimal, but it does have a huge impact if I say, 'Well you have a high BP and you need medication for it'. I think in some cases I am inclined to say to myself, 'Well then just leave it' [don't start AHM]. (GP 3, male, 5-10 years' experience as GP)
Table 2 Main barriers and enablers to start, intensify, continue or deprescribe antihypertensive medication

\begin{tabular}{|c|c|}
\hline Enabler & Barrier \\
\hline \multicolumn{2}{|l|}{ Starting AHM } \\
\hline High SBP (> $180 \mathrm{~mm} \mathrm{Hg}$ ) & Age $>80$ years \\
\hline History of CVD/DM & Limited life expectancy \\
\hline \multicolumn{2}{|l|}{ Planned operation } \\
\hline \multirow[t]{2}{*}{ Patient preference } & $\begin{array}{l}\text { Psychological impact of } \\
\text { starting medication }\end{array}$ \\
\hline & Patient preference \\
\hline \multicolumn{2}{|l|}{ Intensifying AHM } \\
\hline $\begin{array}{l}\text { High SBP }(>140 \text { or } \\
>160 \mathrm{~mm} \mathrm{Hg})\end{array}$ & Age $>80$ years \\
\hline Age $<80$ years & $\geq 3$ antihypertensive drugs \\
\hline \multirow[t]{2}{*}{ History of CVD/DM } & Patient preference \\
\hline & Frailty \\
\hline \multicolumn{2}{|l|}{ Continuing AHM } \\
\hline \multicolumn{2}{|l|}{$\begin{array}{l}\text { Automated prescription } \\
\text { routines }\end{array}$} \\
\hline \multicolumn{2}{|l|}{ Time constraints } \\
\hline $\begin{array}{l}\text { Requires less justification } \\
\text { than deprescribing/ } \\
\text { intensifying }\end{array}$ & \\
\hline
\end{tabular}

Anticipating discomfort

when disturbing the

precarious balance

\section{Target BP level not yet reached}

\section{Deprescribing AHM}

\section{Prolonged achievement of Anticipated regret} target BP

Side effects, orthostatic hypotension

Deprescribing may give the impression of giving up on a patient

$\begin{array}{ll}\text { Risk of falling } & \text { AHM gives patients a sense } \\ \text { of control }\end{array}$

\section{Patient preference}

Experience with increase in quality of life

\section{Terminal illness}

AHM, antihypertensive medication; BP, blood pressure; CVD, cardiovascular disease; DM, diabetes mellitus; SBP, systolic blood pressure.

GPs felt more inclined to start AHM in patients with a history of CVD (ie, secondary prevention) or diabetes and in case of a planned operation. GPs were reluctant to start AHM when patients felt resistant towards medication or, on the contrary, were inclined to start AHM when patients were fearful for the consequences of a high BP:

I have an older female who was very afraid for her high BP. She used to have an angiotensin-converting 


\begin{tabular}{|c|c|}
\hline Start AHM & $\begin{array}{l}\text { "We had a woman who just moved into an elderly home and came under our care. This is a woman of } \\
91 \text { years old. She came to live there with her husband, because of her age and because she had mild } \\
\text { dementia. And when she arrived at the home for elderly they immediately measured her BP. She had a } \\
\text { BP of } 190 \text { over } 90 \text {. And so we gave her losartan } 50 \mathrm{mg} \text {. [...] So before that she had no AHM. Well you } \\
\text { may think, that doesn't do much, it isn't that much. And so, we gave it. Then her BP immediately went } \\
\text { to } 140 \text { over } 80 \text { and it remained there. And then she started complaining about terrible dizziness. And } \\
\text { so I stopped it again." (GP 12, female, >15years' experience as GP) }\end{array}$ \\
\hline Continue AHM & $\begin{array}{l}\text { "A patient of } 92 \text { years old, I think. Known with heart failure, poor mobility and COPD. She wants as } \\
\text { little as possible. Also she doesn't want to go to the hospital. And her BP is actually not much of an } \\
\text { issue. Even though we know it is higher from time to time. When the oedema increases, her legs are } \\
\text { swollen and she gets shortness of breath on exertion, well then we always measure the BP to see } \\
\text { how much room we have to increase the furosemide. And there is always enough room, she always } \\
\text { has a BP of } 170-180 \text {. So that is nice, that we have that. But it never crossed my mind, when we have } \\
\text { treated the fluid retention, to follow-up on her BP to say, let's see if we should treat this structurally." } \\
\text { (GP 15, female, 10-15years' experience) }\end{array}$ \\
\hline Deprescribe AHM & $\begin{array}{l}\text { "Here I have the file of a } 69 \text { years old woman who has stage four lung cancer with progressive brain } \\
\text { metastases. [...] Because of a language barrier, her daughter explained that her mother often felt } \\
\text { dizzy when getting up. She first called it vertigo, but after some further questioning it appeared more } \\
\text { like light headedness. [...] Her BP was repeatedly around } 124 \text { over } 70 \text {. And what I did was, first I } \\
\text { stopped the hydrochlorothiazide. Then her BP stayed low and she was still dizzy. And in the end I also } \\
\text { stopped her losartan. [...] And now her BP stays around } 130 \text { over } 80 \text {, but now without any AHM. So in } \\
\text { hindsight I think she was severely over-treated." (GP 11, male, } 0-5 \text { years' experience) }\end{array}$ \\
\hline
\end{tabular}

AHM, antihypertensive medication; BP, blood pressure; COPD, chronic obstructive pulmonary disease; GP, general practitioner.

enzyme (ACE) inhibitor which I stopped, or reduced. I strictly monitored her BP for 6 months; during which it was always good. And then, a few months later, it went up again; systolic (BP) around 190 or 200. And then she became very anxious. So she wanted her ACE-inhibitor back. (GP 1, male,>15 years' experience)

\section{Intensifying treatment}

Intensifying treatment in patients already receiving AHM (ie, increasing dosage and/or supplementing by adding another antihypertensive drug) seemed relatively straightforward. This was proposed for a SBP higher than $140 \mathrm{~mm} \mathrm{Hg}$ in patients younger than 80 years or with a history of CVD or diabetes and for SBP readings higher than $160 \mathrm{~mm} \mathrm{Hg}$ in patients older than 80 years. GPs experienced more uncertainty when patients already used at least three antihypertensive drugs or when patients wished to refrain from intensifying treatment:

I often see that people do not want this much medication. That they just want to feel comfortable at home. Then (at older ages) you will look more at the person behind the illness. (GP 5, male, >15 years' experience)

Another reason not intensify treatment was frailty:

(Interviewer: How does frailty influence your choice with regard to AHM?) That your target $\mathrm{BP}$ is less strict. That you will accept a BP of 165 over 95 and not give someone extra medication. While in the fit older patient you will think, let's give it a go (increase AHM). (GP 2, female, 10-15 years' experience)

\section{Continuation of treatment}

Continuation of AHM with unchanged regimens was often not an active choice but a consequence of automated repeat prescription algorithms in the electronic health record. In combination with time constraints within the general practice organisation, this prompted swift, global checks of AHM regimens only without room for an assessment whether the prescribed medication was still appropriate. In some cases, GPs thought it might have been better if they deprescribed AHM; however, they felt that leaving the drug regimen unaltered would require less justification than changing things around:

I also think that it is easier to simply continue a treatment. I feel that changing a treatment requires much more justification and should also be done in consultation with the patient or family. Sometimes it can be easier to just consent to a repeat prescription, than to repeatedly consider whether it is still appropriate. (GP 8, male, 0-15 years' experience)

When continuation of AHM was an active choice, an important motivation for leaving things as they were was the perception that some patients had found a precarious balance and changing AHM (ie, increasing or decreasing) could increase the risk of discomfort: 
And reducing, well I think that... it's like 'never change a winning team'. If someone is doing well and has no complaints, then you are not inclined to...(change AHM). (GP12, female, >15 years' experience)

Finally, one GP thought that aiming for a certain BP target also contributed to the steady state in repeat AHM prescriptions, which kept her from regularly re-evaluating whether they were still appropriate:

I think that, if I look at my experience, when you have made the decision to act on a BP, I think that you are more inclined to continue your aim for the target BP that you have set. [...] I think that you tend to just proceed and not use every evaluation moment to reconsider whether you should continue with it (treatment). Which really would have been very appropriate. But I notice that I do not do that myself. (GP 15, female, 5-10 years' experience)

\section{Deprescribing treatment}

Reducing or discontinuing AHM was not something that was easily decided on. It required clear motives apart from a prolonged period during which the BP was at or below target level, including side effects of AHM, orthostatic hypotension, a high risk of falling and patient preference. Some GPs had experience with an increase in quality of life (for example feeling less tired) of patients after deprescribing AHM:

When I stop AHM in older patients they often feel much better. Then the BP rises somewhat and they feel less tired. Well I don't know if it is caused by that, but sometimes I think that. (GP 1, male, $>15$ years' experience)

In some cases, the decision of GPs was influenced by anticipated regret: the fear that a patient might have a stroke after deprescribing AHM:

And it requires a sort of courage to notice that when you reduce AHM, the values, the indicators, increase. And you have to feel comfortable with that. If you withdraw AHM and someone, say after six months, has a stroke, than you suddenly feel uncomfortable. (GP 5, male, >15 years' experience)

GPs acknowledged that in the terminal phase it would be rational to discontinue AHM. However, they often hesitated to take this step to avoid the impression they were giving up on the patient or unnecessarily deprive them of a sense of being in control with adequate BP measurements. They aimed to avert the risk of non-fatal stroke and the accompanying functional limitations in the last phase of life.

\section{Current guideline and future support}

During the interview, when provided with ample time to reflect on decisions that had been made, the interviewed GPs came to new insights and ideas. They stated that in daily practice treatment decisions were often based on intuition and that it might have been better if they had deprescribed AHM earlier:

I often do this briefly in between my consultations. And I notice, now that we are talking about it... it forces you to critically think about it. And then I think, oh I could do this better. And of course we can always do our work better; nobody knows everything. But I do think that, now that you mention this and we critically look at it, I should think this through more thoroughly. (GP 11, male, 0-5 years' experience)

GPs generally felt insufficiently supported by the guidelines in their efforts to treat hypertension in older people:

Yes, whom to treat and whom not to treat among the oldest old. [...] The healthy you have to treat, because they still have a long life expectancy and therefore have much to gain in lowering their BP. Those with diseases, like the patients with diabetes or a myocardial infarction, you have to treat because they have a substantially elevated risk because of their disease that we are well able to bring down. That group in between, with frail elderly, I followed the advice not to treat them for a while. And now I recently heard that you also have to treat those. I just don't know it anymore. I would really like to have a guideline that states: in elderly you have pay attention to this, this and this. (GP 9, female, 5-10 years' experience)

A GP suggested a treatment flow diagram including more subjective factors like frailty for future support or indicators to start or deprescribe AHM like the Screening Tool of Older Person's Prescriptions (STOPP)/Screening Tool to Alert doctors to Right Treatment criteria. ${ }^{13}$ Another suggestion was to aid deprescription by incorporating algorithms in the electronic health record that aid doctors and practice nurses in observing complaints that are potentially related to a low BP.

\section{DISCUSSION}

For Dutch GPs, starting or intensifying antihypertensive medication is guided by the absolute level of SBP, history of CVD and diabetes. GPs feel reluctant to start AHM at older age, but when someone already receives AHM they also feel reticent in accepting a high BP (ie, not intensifying AHM). They may refrain from intensifying AHM in cases of frailty or clear patient preference. There is a propensity to continue AHM in older people within a daily routine that insufficiently supports critical review of prescriptions due to time constraints and automated prescription routines. Continuation of AHM is also supported by anticipating discomfort when disturbing patients' delicate balance and the perception that continuing treatment requires less justification. The decision to deprescribe AHM is difficult, although GPs also mention a possible gain in quality of life, such as less fatigue. Deprescription is only considered when BP is consistently at or below the 
Box 2 Antihypertensive treatment in Dutch general practices

- Almost all Dutch citizens are registered at a general practice.

- In the Netherlands, general practitioners (GPs) have a gatekeeping role and are relatively easy accessible. ${ }^{28}$

- Approximately $75 \%-80 \%$ of medication is therefore prescribed by $\mathrm{GPs}^{29}$

- In 2006, the guideline on hypertensive treatment was combined into a more general guideline on cardiovascular risk management (CVRM). ${ }^{30}$

- Recommendations in this guideline are similar to the European guideline, aside from the Systematic Coronary Risk Estimation chart which is expanded up to 70 years and the target systolic blood pressure for people over 80 years old which is $150-160 \mathrm{~mm} \mathrm{Hg}{ }^{2}$

- With the introduction of nurse-led CVRM, over the last decade, the workload for GPs decreased and guideline adherence improved. ${ }^{31}$

- Specialists are mainly responsible for starting antihypertensive treatment in patients with secondary prevention, while general practitioners play a crucial part in primary prevention.

target, and a patient experiences side effects or wants to reduce treatment. Anticipated regret of a future stroke may represent an additional barrier for deprescription. GPs experience insufficient guidance on antihypertensive treatment for older people and would welcome a treatment flow diagram including specific STOPP criteria as well as more subjective factors like frailty.

An important strength of our study is the diversity of GPs that were interviewed, with a broad range in experience and setting. The interview guide was aimed at discussing examples from daily clinical practice to limit the chance of socially desirable answers. We followed the consolidated criteria for reporting qualitative research guidelines to improve the interpretation and reproducibility of our results. ${ }^{14}$ A limitation is that our study only includes Dutch GPs, which may restrict the level of generalisability. The role of Dutch practice nurses within the cardiovascular prevention programmes has become more prominent over the last years (box 2).$^{15}$ This probably limits the time Dutch GPs spend on antihypertensive treatment and may facilitate routine continuation of AHM. Since specific directives on BP treatment for older people are lacking in most international guidelines, the feeling of limited support is likely found in other countries as well. Relative few patient cases were discussed were AHM was started due to the reluctance of GPs to start AHM in older patients and the notion that a lot of the older patients already received AHM. Even though data saturation was reached, this could have potentially limited the scope of reasons to start AHM.

No other qualitative study has assessed GPs' reasons for prescription and deprescription of antihypertensive medication in older patients. There are however previously reported studies with a broader focus on cardiovascular prevention, which have shown results consistent to ours. ${ }^{16-18}$ These studies stated that GPs are uncertain about many aspects of cardiovascular prevention, including the application of guidelines, organisation of care and benefit for individual patients and that they are less likely to prescribe preventive medication in frail older people. ${ }^{31617}$ Especially when focusing on AHM, the uncertainties are most likely fuelled by the conflicting available evidence. There is both evidence in favour of intensifying treatment as well as studies that support a higher target BP. $^{610}$ These uncertainties may lead to overtreatment by unnecessary continuation of AHM regimens, or to undertreatment, caused by the reluctance to initiate treatment in older people who are still free from AHM. As continuation also seems to be a consequence of daily routine influenced by time constraints and automated prescription routines, overtreatment might be prevented by more structural time for medication reviews. Patient preference plays a crucial part in (de)prescribing and should be actively taken into consideration, for example through use of the outcome prioritisation tool. ${ }^{19}$ In addition, an estimation of frailty seems important which, by its subjective nature, could bring about the large variation in treatment intentions of GPs, which was previously described in a Belgian vignette study. ${ }^{20}$

The reluctance to deprescribe AHM in older patient is also noted for other drug classes. ${ }^{21}$ This reluctance is, among other factors, induced by GPs' anticipated regret, which has been previously described as a motivator to start or continue preventive treatment. ${ }^{1722}$ The fear of 'causing' a stroke by deprescribing AHM is unsupported by clinical evidence but might be instigated by results from trials like the SPRINT trial that support intensive BP lowering, ${ }^{6}$ even though multimorbid older people are potentially under-represented in this trial and the generalisability of these results is uncertain. ${ }^{8}$ Also for deprescribing shared decision-making is crucial. ${ }^{21}$ Older patients have varied attitudes and ideas about their medication use and clear communication is therefore crucial. ${ }^{23}$ However, given the present knowledge gaps, risk communication is perceived difficult by GPs. ${ }^{24}$ Until now, deprescribing AHM is only supported by observational studies, which have demonstrated an association between a low or decreasing BP and worse functional outcome, cognitive decline and increased mortality. ${ }^{25}{ }^{26}$ The Discontinuation of Antihypertensive Treatment in Elderly People trial showed that discontinuation of AHM was safe, although not beneficial on cognitive, psychological or general daily functioning during the relatively short 16 -week follow-up. ${ }^{27}$ To make a better informed decision on the deprescription of AHM, it would be useful to have additional evidence on the efficacy of deprescription with longer follow-up and on the subjective and objective impact of AHM in older people. For GPs, it would be desirable to add indicators for deprescription (ie, STOPP criteria) in the guideline as this is what GPs consider difficult.

Dutch GPs are reluctant to start or deprescribe AHM in older people. Continuation of AHM is reinforced by a daily routine that insufficiently supports critical medication review and conflicting available evidence on efficacy of intensifying treatment. Patient preference appears 
crucial for both prescribing and deprescribing. Anticipated regret of a future stroke works as a barrier for deprescription. Current guidelines provide insufficient guidance and clear indicators on deprescription could endorse it.

Acknowledgements We would like to thank all general practitioners who participated in the interviews for their valuable input.

Contributors TvanM: study design, data acquisition, data analysis, data interpretation, drafting the manuscript. SDI: data acquisition, data analysis, data interpretation, critical revision of manuscript. PGvanP and RKEP: data interpretation, critical revision of manuscript. ER, AJP and EMvanC: study design, data interpretation, critical revision of manuscript.

Funding The research leading to these results has received funding from the European Union Seventh Framework Programme (FP7/2007-2013) under grant agreement no. 305374 and an EU Joint Programme-Neurodegenerative Disease Research (JPND) project. The JPND project is supported through the following funding organisations under the aegis of JPND—www.jpnd.eu: Finland, Suomen Akatemia (Academy of Finland,291803); France, L'Agence Nationale de la Recherche (The French National Research Agency, ANR-14-JPPS-0001-02); Germany, Bundesministerium für Bildung und Forschung (BMBF) (The Federal Ministry of Education and Research, FKZ01ED1509); Sweden, Vetenskapsrådet (VR) (Swedish Research Council, 529-2014-7503), The Netherlands, ZonMw (The Netherlands Organization for Health Research and Development, 733051041).

Competing interests None declared.

Patient consent Obtained.

Provenance and peer review Not commissioned; externally peer reviewed.

Data sharing statement Data will not be made publicly available. For more information, please contact the corresponding author.

Open Access This is an Open Access article distributed in accordance with the Creative Commons Attribution Non Commercial (CC BY-NC 4.0) license, which permits others to distribute, remix, adapt, build upon this work non-commercially, and license their derivative works on different terms, provided the original work is properly cited and the use is non-commercial. See: http://creativecommons.org/ licenses/by-nc/4.0/

(C) Article author(s) (or their employer(s) unless otherwise stated in the text of the article) 2018. All rights reserved. No commercial use is permitted unless otherwise expressly granted.

\section{REFERENCES}

1. Gu A, Yue Y, Argulian E. Age Differences in Treatment and Control of Hypertension in US Physician Offices, 2003-2010: A Serial Crosssectional Study. Am J Med 2016;129:50-8.

2. Piepoli MF, Hoes AW, Agewall S, et al. 2016 European Guidelines on cardiovascular disease prevention in clinical practice: The Sixth Joint Task Force of the European Society of Cardiology and Other Societies on Cardiovascular Disease Prevention in Clinical Practice (constituted by representatives of 10 societies and by invited experts) Developed with the special contribution of the European Association for Cardiovascular Prevention \& Rehabilitation (EACPR). Eur Heart $J$ 2016;37:2315-81.

3. Streit S, Verschoor M, Rodondi N, et al. Variation in GP decisions on antihypertensive treatment in oldest-old and frail individuals across 29 countries. BMC Geriatr 2017;17:93.

4. Bejan-Angoulvant T, Saadatian-Elahi M, Wright JM, et al. Treatment of hypertension in patients 80 years and older: the lower the better? A meta-analysis of randomized controlled trials. $J$ Hypertens 2010;28:1366-72.

5. Wright JT, Williamson JD, Whelton PK, et al. A Randomized Trial of Intensive versus Standard Blood-Pressure Control. N Engl J Med 2015;373:2103-16.

6. Williamson JD, Supiano MA, Applegate WB, et al. Intensive vs Standard blood pressure control and cardiovascular disease outcomes in adults aged $\geq 75$ years: a randomized clinical trial. JAMA 2016;315-2673-82.

7. Marcum ZA, Perera S, Newman AB, et al. Antihypertensive Use and Recurrent Falls in Community-Dwelling Older Adults: Findings From the Health ABC Study. J Gerontol A Biol Sci Med Sci 2015;70-1562-8.
8. Supiano MA, Williamson JD. Applying the Systolic Blood Pressure Intervention Trial Results to Older Adults. J Am Geriatr Soc 2017;65:16-21

9. Chobanian AV. SPRINT Results in older patients: how low to go? JAMA 2016;315:2669-70.

10. Muller M, Smulders YM, de Leeuw PW, et al. Treatment of hypertension in the oldest old: a critical role for frailty? Hypertension 2014;63:433-41.

11. Giacomini MK, Cook DJ. Users' guides to the medical literature: XXIII. Qualitative research in health care A. Are the results of the study valid? Evidence-Based Medicine Working Group. JAMA 2000;284:357-62.

12. Braun V, Clarke V. Using thematic analysis in psychology. Qual Res Psychol 2006;3:77-101

13. O'Mahony D, O'Sullivan D, Byrne S, et al. STOPP/START criteria for potentially inappropriate prescribing in older people: version 2. Age Ageing 2015;44-213-8.

14. Tong A, Sainsbury P, Craig J. Consolidated criteria for reporting qualitative research (COREQ): a 32-item checklist for interviews and focus groups. Int J Qual Health Care 2007;19:349-57.

15. Schäfer WL, Boerma WG, Spreeuwenberg P, et al. Two decades of change in European general practice service profiles: conditions associated with the developments in 28 countries between 1993 and 2012. Scand J Prim Health Care 2016;34-97-110.

16. Drewes YM, Koenen JM, de Ruijter W, et al. GPs' perspectives on preventive care for older people: a focus group study. $\mathrm{Br} J$ Gen Pract 2012;62:765-72.

17. van Peet PG, Drewes YM, Gussekloo J, et al. GPs' perspectives on secondary cardiovascular prevention in older age: a focus group study in the Netherlands. Br J Gen Pract 2015;65:e739-47.

18. Jansen J, McKinn S, Bonner C, et al. General Practitioners' Decision Making about Primary Prevention of Cardiovascular Disease in Older Adults: A Qualitative Study. PLoS One 2017;12:e0170228.

19. van Summeren JJ, Schuling J, Haaijer-Ruskamp FM, et al. Outcome prioritisation tool for medication review in older patients with multimorbidity: a pilot study in general practice. $\mathrm{Br} J$ Gen Pract 2017;67:e501-6.

20. Mermans E, Degryse J, Vaes B. Treatment intentions of general practitioners regarding hypertension in the oldest old: a vignette study. BMC Fam Pract 2016;17:122.

21. Jansen J, Naganathan V, Carter SM, et al. Too much medicine in older people? Deprescribing through shared decision making. BMJ 2016;353:i2893.

22. Luymes $\mathrm{CH}$, Boelhouwer NJ, Poortvliet RK, et al. Understanding deprescribing of preventive cardiovascular medication: a Q-methodology study in patients. Patient Prefer Adherence 2017;11:975-84.

23. Weir K, Nickel B, Naganathan V, et al. Decision-Making Preferences and Deprescribing: Perspectives of Older Adults and Companions About Their Medicines. J Gerontol B Psychol Sci Soc Sci 2017 (accessed 12 Jan2017).

24. Schuling J, Gebben H, Veehof LJ, et al. Deprescribing medication in very elderly patients with multimorbidity: the view of Dutch GPs. A qualitative study. BMC Fam Pract 2012;13:56.

25. Boutitie F, Gueyffier F, Pocock S, et al. J-shaped relationship between blood pressure and mortality in hypertensive patients: new insights from a meta-analysis of individual-patient data. Ann Intern Med 2002;136:438-48

26. Ogliari G, Westendorp RG, Muller M, et al. Blood pressure and 10year mortality risk in the Milan Geriatrics $75+$ Cohort Study: role of functional and cognitive status. Age Ageing 2015:44:932-7.

27. Moonen JE, Foster-Dingley JC, de Ruijter W, et al. Effect of discontinuation of antihypertensive treatment in elderly people on cognitive functioning--the DANTE Study Leiden: a randomized clinical trial. JAMA Intern Med 2015;175:1622-30.

28. van den Berg MJ, van Loenen T, Westert GP. Accessible and continuous primary care may help reduce rates of emergency department use. An international survey in 34 countries. Fam Pract 2016;33:42-50.

29. Ohlsson H, Vervloet M, van Dijk L. Practice variation in a longitudinal perspective: a multilevel analysis of the prescription of simvastatin in general practices between 2003 and 2009. Eur J Clin Pharmacol 2011;67:1205-11.

30. Wiersma T, Smulders YM, Stehouwer CD, et al. [Summary of the multidisciplinary guideline on cardiovascular risk management (revision 2011)]. Ned Tijdschr Geneeskd 2012;156:A5104.

31. Voogdt-Pruis HR, Van Ree JW, Gorgels AP, et al. Adherence to a guideline on cardiovascular prevention: a comparison between general practitioners and practice nurses. Int J Nurs Stud 2011;48:798-807. 